\title{
La discapacidad laboral de las madres se asocia con Depresión Mayor entre los adolescentes
}

\author{
Sirkka Peiponen* ML \\ Eila Laukkanen* MD \\ Veijo Korhonen* ML \\ Johannes Lehtonen ${ }^{\star \star} M D$ \\ * Psiquiatra Infantojuvenil. Hospital \\ Universitario de Kuopio \\ ** Profesor. Departamento de Psiquiatría. \\ Hospital Universitario de Kuopio y \\ Universidad de Kuopio \\ FINLANDIA
}

\begin{abstract}
RESUMEN - Objetivos: En este trabajo se investigan los factores familiares y sociodemográficos asociados a un Trastorno Depresivo Mayor entre adolescentes en tratamiento ambulatorio.

Metodología: Se han comparado adolescentes con Trastorno Depresivo Mayor $(\mathrm{n}=18)$ con otros con Trastorno Depresivo Mayor y patología psiquiátrica comórbida $(n=25)$ y con otro grupo de adolescentes sin patología psiquiátrica de ningún tipo $(\mathrm{n}=28)$. El diagnóstico se hizo mediante la Entrevista Clínica Estructurada de la DSM-III (SCID). En esos tres grupos se investigaron la situación de los padres en el trabajo, el tipo de trabajo y el status marital de los padres, así como la situación económica de la familia y sus necesidades de ayudas públicas.

Resultados: La discapacidad laboral de la madre estaba significativamente asociada con depresión y patología psiquiátrica. No se encontraron en los diferentes grupos estudiados ningún otro factor familiar o sociodemográfico asociado a depresión.

Conclusiones: Es importante investigar el contexto familiar de los adolescentes depresivos, especialmente la situación de la madre en su trabajo ya que estos factores pueden afectar a la gravedad de la enfermedad depresiva. Por ello en el tratamiento de estos enfermos se deben tener en cuenta los aspectos clínicos y preventivos en relación con estas discapacidades laborales de la madre.
\end{abstract}

\section{Introducción}

Los estudios realizados sobre la comorbilidad psiquiátrica en la adolescencia, muestran como se ven afectadas importantes areas de la personalidad (Lewinsohn, Rohde, Seeley 1995). Entre los adolescentes la enfermedad depresiva está asociada a un gran número de episodios depresivos e intentos de suicidio pre- 
vios, así como a una gran demanda de servicios de salud mental (Rohde, Lewinsohn, Seeley 1991). Los factores asociados con la comorbilidad psiquiátrica en el caso de la depresión del adolescente, poseen un particular interés al estar este tipo de depresión con comorbilidad generalmente asociado con mas problemas psiquiátricos graves que lo que sucede en el caso de otras formas de depresión.

Estudios anteriores han mostrado como el ambiente familiar, las relaciones sociales en la familia, los diferentes acontecimientos vitales de carácter adverso y factores estresantes, constituyen factores de riesgo en el desarrollo de una depresión durante la adolescencia (Prange et al. 1992; Olsson 1998; Goodman et al. 1998; Lewinsohn et al. 1994), sin embargo existe muy poca información acerca de los factores que se asocian con la enfermedad depresiva y con la patología psiquiátrica comórbida.

El objeto de este estudio es determinar si en una muestra de adolescentes enviados para tratamiento psiquiátrico ambulatorio se dan diferencias en los contextos sociodemográficos parentales según los sujetos padezcan un Trastorno Depresivo Mayor, un Trastorno Depresivo Mayor con patología psiquiátrica comórbida, o no sufran patología psiquiátrica alguna.

\section{Sujetos y métodos}

Los sujetos de este estudio son adolescentes que habían solicitado ayuda a lo largo de un año en las Consultas Externas de Psiquiatría de Adolescentes del Hospital Universitario de Kuopio. Aceptaron participar en el estudio un total de 164 adolescentes. Los padres de 97 de ellos fueron entrevistados como parte de la valoración general,pero 67 no fueron entrevistados por las siguientes razones: 46 adolescentes no dieron permiso para que sus padres fue- sen entrevistados; los padres de 8 adolescentes no acudieron a la entrevista cuando se les citó a ella; los padres de 2 adolescentes rechazaron tomar parte en el estudio; los padres de 6 vivían demasiado lejos como para acudir a la entrevista; en 2 casos se estaba en plena crisis marital o psiquiátrica lo cual no hizo posible la entrevista; y en 3 casos no se nos ofreció ninguna razón para no tenerla. Hay que señalar que los padres de 93 adolescentes eran sus padres biológicos, mientras que en 4 casos se trataba de padres adoptivos. Padre y madre fueron entrevistados en la totalidad de los casos, excepto en 4 en los que asistió solo el padre y en 25 en los que vino sola la madre.

La aprobación para llevar a cabo este estudio fue obtenido del Comité Etico del Hospital Universitario de Kuopio y de la Universidad de Kuopio.

El diagnóstico psiquiátrico se hizo mediante el empleo de la Entrevista Clinica Estructurada de la DSM-III-R (SCID) (Riskind et al. 1987, Skre et al. 1991, Spitzer et al. 1992, Williams et al. 1992) El item correspondiente a los Trastornos de la Conducta de la SCID II se combinó con items de la SCID I, y las valoraciones fueron realizadas por cuatro experimentados psiquiatras de adolescentes entrenados en la utilización del SCID I.

Los sujetos del estudio no estaban presentes cuando sus padres eran entrevistados po profesionales bien experimentados que trabajaban en las consultorios externos ambulatorios. Cada entrevista fue realizada siguiendo un cuestionario semiestructurado que incluye preguntas acerca del contexto familiar, acontecimientos vitales y relaciones familiares. Las variables investigadas en este estudio fueron divorcio de los padres, conflictos maritales graves (Padres que han vivido separados durante el periodo adolescente de su hijo debido ello a discordias maritales), muerte de algún padre, desempleo de los 
padres, discapacidad laboral de los padres debida a enfermedad, problemas económicos en la familia (Experiencia subjetiva de los padres) y pertenencia durante el año previo a la entrevista a algún sistema de cobro de subsidio económico de carácter público.

La muestra final ( $\mathrm{n}=71$ de los que el $28 \%$ eran chicos) estaba compuesta por 1) Adolescentes sin diagnóstico psiquiátrico $(\mathrm{n}=28$ con $32 \%$ de chicos). 2) Adolescentes con Trastorno Depresivo Mayor solo ( $\mathrm{n}=18$ con un $24 \%$ de chicos). 3) Adolescentes con Trastorno Depresivo Mayor y patología psiquiátrica comórbida ( $n=25$ con un $28 \%$ de chicos). El grupo de adolescentes con otros diagnósti$\cos (n=26)$ fue excluido de este estudio dada la heterogeneidad del diagnóstico.

Los diagnósticos psiquiátricos comórbidos fueron: Trastornos de Ansiedad (Williams et al. 1992), Distimias (Olsson 1998), Dependencia alcohólica (Kaslow et al. 1994), Trastornos de la Conducta (Goodman et al. 1998) y Anorexia (Goodman et al. 1998). Cinco adolescentes recibieron mas de un diagnóstico comórbido.

\section{Resultados}

La edad media de los adolescentes no difiere significativamente entre los grupos estudiados (Sin diagnóstico 16,7 +/- 1,83, rango 13,5 a 20,8; Trastorno Depresivo Mayor solo 17,1 +/- 2,13, rango 13,7 a 20,9; Trastorno Depresivo Mayor con patología comórbida 17,1 +/1,95, rango 13,9 a 21,4). La media de edad de las madres fue de 43,4 +/- 4,53 años, rango 31 a 56 años, y la de los padres fue de $46,4+/-$ 5,42 años, rango 34 a 62 años.

Las madres de los adolescentes con enfermedad psiquiátrica comórbida estaban laboralmente discapacitadas mas a menudo de lo que sucedía en el caso de las otras madres (tabla I). El porcentaje de problemas psicosociales entre los padres de la muestra estudiada era alto, por ejemplo los divorcios suponían el $32 \%$ de los casos. Los problemas económicos eran comunes en todos los grupos y la proporción de familias que recibían algún tipo de asistencia pública era alta, especialmente entre el grupo de adolescentes con Trastorno Depresivo Mayor y enferme-

Tabla I

Contexto sociodemográfico familiar de adolescentes con Trastorno Depresivo Mayor, con Trastorno Depresivo Mayor y patología comórbida y sin diagnóstico de enfermedad psiquiátrica

\begin{tabular}{|c|c|c|c|c|c|}
\hline Variable & $\begin{array}{c}\text { Trastorno } \\
\text { Depresivo } \\
\text { Mayor } \\
(n=25)\end{array}$ & $\begin{array}{c}\text { Trastorno } \\
\text { Depresivo } \\
\text { Mayor } \\
(\mathrm{n}=18)\end{array}$ & $\begin{array}{l}\text { Sin diagnóstico } \\
\text { psiquiátrico } \\
(\mathrm{n}=28)\end{array}$ & Todo & Significación ${ }^{1}$ \\
\hline \multicolumn{6}{|l|}{ La madre sufre de } \\
\hline discapacidad laboral (\%) & 24 & 0 & 4 & 10 & $\mathrm{p}<0.05$ \\
\hline \multicolumn{6}{|l|}{ El padre sufre de } \\
\hline discapacidad laboral $(\%)$ & 20 & 5 & 14 & 14 & n.s. \\
\hline La madre está desempleada (\%) & 12 & 5 & 4 & 7 & n.s. \\
\hline El padre está desempleado (\%) & 8 & 5 & 7 & 7 & n.s. \\
\hline Problemas económicos en la familia (\% & \%) 64 & 50 & 50 & 55 & n.s. \\
\hline \multicolumn{6}{|c|}{ La familia recibe asistencia } \\
\hline económica pública (\%) & 28 & 5 & 14 & 17 & n.s. \\
\hline Muerte parental $(\%)$ & 8 & 17 & 7 & 10 & n.s \\
\hline Grave discordia marital $(\%)$ & 16 & 11 & 14 & 14 & n.s. \\
\hline Divorcio parental (\%) & 36 & 28 & 32 & 32 & n.s. \\
\hline
\end{tabular}

1. Test del Chi cuadrado y t-test exacto de Fischer. 
dad comórbida. No obstante los grupos diagnósticos no difieren significativamente uno del otro en este aspecto, posiblemente debido al pequeño tamaño de la muestra.

\section{Discusión}

Existen muchos trabajos que sugieren que los variados problemas de índole familiar predisponen al padecimiento de depresiones y otros trastornos psiquiátricos entre los adolescentes (Prange et al. 1992, Olsson 1998, Lewinsohn et al. 1994, Palosaari,Aro 1995).

Estudios previos han mostrado como casi la mitad de los adolescentes con Trastorno Depresivo Mayor también poseen un riesgo de padecimiento de otros trastornos mentales a lo largo de sus vidas (Lewinsohn, Rohde, Seeley 1998). El grado de comorbilidad parece ser mas elevado entre los adolescentes que entre los adultos (Rohde, Lewinsohn, Seeley 1991). La aparición de mas de un trastorno psiquiátrico tiene graves y negativas consecuencias en el funcionamiento existencial de estos adolescentes, afectándoles en su rendimiento académico y necesitando mas frecuentemente tratamiento de sus problemas de salud mental, habiendo presentado además intentos de suicidio previos (Lewinsohn, Rohde, Seeley 1995; Lewinsohn, Rohde, Seeley 1998).

En nuestro estudio fueron diferentes los problemas sociodemográficos presentes entre las familias de estos adolescentes que habían sido enviados a los servicios ambulatorios psiquiátricos. El único factor específico sociodemográfico de las familias asociado con Trastorno Depresivo Mayor y patología psiquiátrica comórbida fue la discapacidad laboral de la madre. Los adolescentes de todos los grupos tenían problemas familiares variados, pero solamente la discapacidad laboral de la madre se asociaba significativamente con la Depresión y ello se dio solo en el grupo con patología psiquiátrica comórbida. Es bien conocido que la depresión parental constituye un factor predisponente para la depresión del adolescente (Kaslow, Deering, Racusin 1994; Shiner, Marmorstein 1998). El papel de la madre para garantizar la seguridad y el funcionamiento diario de la familia puede ser especialmente importante con respecto a ello, pero la realidad es que son necesarias futuras investigaciones.

Es posible que los problemas económicos y el desempleo puedan también estar asociados con la Depresión en los adolescentes, aunque probablemente no con la misma importancia que lo que sucede con la discapacidad de la madre. Su efecto no apareció como significativo en nuestra muestra, pero ello puede ser debido al tamaño pequeño de la misma y al potencial error estadístico tipo 2 .

La información relativa al contexto familiar en el caso de los adolescentes con depresión es importante para los planes de tratamiento de los mismos (Hammen et al. 1998) así como para establecer la necesaria cooperación con los padres, cosa que es verdaderamente útil para la valoración y el tratamiento de los pacientes adolescentes (Lewinsohn, Rohde, Seeley 1998). La exis tencia de unos lazos familiares sólidos y positivos constituye un factor protector contra los riesgos de padecer cuadros psiquiátricos entre los adolescentes (Resnick et al. 1997). La cercanía a los padres protege la autoestima de los adolescentes hasta en el caso de las situaciones de divorcio parental (Palosaari, Aro 1995; Palosaari, Aro, Laippala 1996). La afectación del funcionamiento psicosocial de la madre debilita su disponibilidad emocional, disminuyendo los sentimientos de cercanía y distorsionando la influencia protectora de las buenas relaciones madre-hijo tan necesarias para el nor- 
mal crecimiento e individuación en el curso del desarrollo del adolescente.

Nuestros resultados apoyan la importancia que tiene el buen funcionamiento materno sobre el conjunto de la familia. No obstante, esto no significa que otros miembros de la familia no sean importantes para el crecimiento y desarrollo del adolescente o para la prevención de la enfermedad depresiva. La discapacidad de la madre puede tener profundos efectos sobre los adolescentes, en mayor modo que otros factores psicosociales, en lo que concierne a generar problemas de salud mental. Claramente parecen ser necesarias nuevas investigaciones en este campo a fin de aclarar con mas detalle los diferentes papeles ejercidos por los diferentes miembros de la familia en relación con los problemas psiquiátricos de la adolescencia.

\section{Bibliografía}

GOODMAN, S.H., HOVEN, C.W., NARROW, S.E., COHEN, P.,FIELDING, B.M.,ALEGRIA,M.,LEAF, P.J., KANDEL, D., McCUE HORWITZ, S., BRAVO, M., MOORE,R.,DULCAN, M.K. Measurement of risk for mental disorders and competence in the National Health Institute of Mental Health Methods for the Epidemiology of Child and Adolescent Mental Disorders (MECA) study. Social Psychiatry Psychiatry Epidemiology, 33:162-183,1998.

HAMMEN, C., RUDOPH, K., WEISZ, J., RAO, U., BURGE, D. The context of depression in clinic-referred youth:neglected areas in treatment. J Am Acad Child Ado lescent Psychiatry 38: 64-71, 1998.

KASLOW, N.J., DEERING, C.G., RACUSIN, G.R. Depressed children and their families. Clinical Psychology Rev 14: 39-59, 1994.

LEWINSOHN, P.M., ROBERTS, R.E., SEELEY, J.R., ROHDE, P.,GOTLIB, I.H.,HOPS, H. Adolescent psychopathology. II: psychosocial risk factors for depression. Journal Abnormal Psychology 103: 302-315, 1994b.

LEWINSOHN, P.M., ROHDE, P., SEELEY, J.R. Adolescent psychopathology III. The clinical consequences of comorbidity. J Am Acad Child Adolescent Psychiatry 34: 510-519, 1995.

LEWINSOHN, P.M., ROHDE, P.,SEELEY, J.R. Major depressive disorder in older adolescents: prevalence, risk factors and clinical implications. Clinical Psychology Rev 18: 765-794, 1998.

OLSSON, G. Adolescent depression. Epidemiology, nosology, life stress and social network. Minireview based on doctoral thesis. Ups J Med Sci 103: 77-145, 1998.

PALOSAARI, U.K.,ARO, H.M. Parental divorce, selfesteem and depression: an intimate relationship as a protective factor in young adulthood. Journal Affective Disease 35: 91-96, 1995.

PALOSAARI, U., ARO, H., LAIPPALA, P. Parental divorce and depression in young adulthood: adolescents closeness to parents and self-esteem as mediating factor. Acta Psychiatrica Scandinavica 93: 20-36, 1996.

PRANGE, M.E., GREENBAUM, B.E., SILVER, S.E., FRIEDMAN, R.M.,KUTASH, K., DUCHNOWSKI,A.J. Family functioning and psychopathology among adolescents with severe emotional disturbances. Journal Abnor mal Child Psychology 20: 83-102, 1992.

RESNICK, M.D., BEARMAN, P.S., BLUM, R.W., BAUMAN, K.E., HARRIS, K.M., JONES, J., TABOR, J., BEUHRING, T.,SIEVING, R.E.,SHOW, M., IRELAND, M.,BEARINGER,L.H.,UDRY, J.R. Protecting adolescents from harm. Findings from the National Longitudinal Study on Adolescent Health. JAMA 10: 278: 823-32, 1997.

RISKIND, J.H.,BECK,A.T.,BERCHICK,R.J.,BROWN, G., STEER, R.A. Reliability of DSM-III diagnoses for major depression and generalized anxiety disorder using the structural clinical interview for DSM-III. Archives General Psychiatry 44:817-20,1987.

ROHDE, P.,LEWINSOHN, P.M.,SEELEY, J.R. Comorbidity of unipolar depression: II. Journal Abnormal Psycho logy 100: 214-222,1991.

SHINER, R.L., MARMORSTEIN, N.R. Family environments of adolescents with lifetime depression:Associations with maternal depression history. J Am Acad Child Adolesc Psychiatry 37; 11: 1152-1160, 1998.

SKRE, I., ONSTAD, S., TORGERSEN, S., KRINGLEN, E. High interrater realiability for the Structured Clinical Interview for DSM-III-R Axis I (SCID - I). Acta Psychiatrica Scandinavica 84: 167-73, 1991.

SPITZER, R.L.,WILLIAMS, U.B.W., GIBBON, M., FIRST, M.B. The Structured Clinical Interview for DSMIII-R (SCID-I):History, rationale and description. Archives General Psychiatry 9: 624-29, 1992. 
WILLIAMS, B.W.J., GIBBON, M., FIRST, M.B., SPITZER, R.L., DAVIES, M., BORUS, J.,HOWES, M.J., KANE, J.,POPE,H.G., ROUNSAVILLE, B.,WITTCHEN, H.U. The Structured Clinical Interview for DSM-III R (SCID). Archives General Psychiatry 49: 630-6, 1992.
Dirección para correspondencia: Sirkka Peiponen, ML

Department of Psychiatry

Kuopio University Hospital

P.O. Box 1777

FIN-70211 Kuopio

E-mail: sirkka.peiponen@kuh.fi

FINLANDIA 